\begin{tabular}{|c|l|}
\hline Title & Brittle ductile transition of double network hydrogels : Mechanical bal ance of two networks as the key factor \\
\hline Author(s) & A hmed, Saika; Nakajima, Tasuku; Kurokawa, Takay uki; Haque, Md A namul; Gong, Jian Ping \\
\hline Citation & $\begin{array}{l}\text { Polymer, 55(3), 914 923 } \\
\text { https://doi.org/10.1016/.polymer.2013.12.066 }\end{array}$ \\
\hline Issue Date & 2014 02-12 \\
\hline Doc URL & http://hdl.handle.net/2115/55147 \\
\hline Type & article (author version) \\
\hline File Information & Manuscript_final.pdf \\
\hline
\end{tabular}

Instructions for use 


\title{
Brittle-Ductile Transition of Double Network Hydrogels: Mechanical Balance of Two Networks as the Key Factor
}

\author{
Saika Ahmed ${ }^{a}$, Tasuku Nakajima $^{b}$, Takayuki Kurokawa $^{b}$, Md. Anamul Haque, ${ }^{b, \#}$ and Jian Ping \\ Gong ${ }^{\text {,* }}$
}

${ }^{a}$ Graduate School of Life Science, Hokkaido University, Sapporo 060-0810, Japan

${ }^{b}$ Faculty of Advanced Life Science, Hokkaido University, Sapporo 060-0810, Japan

"Present address: Department of Chemistry, University of Dhaka, Dhaka-1000, Bangladesh

* Corresponding author

Tel. \& Fax: +81-11-706-2774, E-mail: gong@mail.sci.hokudai.ac.jp

Key words: Double network hydrogels; fracture; toughness 


\begin{abstract}
Tough double network (DN) hydrogels are a kind of interpenetrating network (IPN) gels with a contrasting structure; it consists of a rigid and brittle $1^{\text {st }}$ network with dilute, densely cross-linked short chains and a soft and ductile $2^{\text {nd }}$ network with concentrated, loosely cross-linked long chains. In this work, we focus on how the brittle gel changes into a tough one by increasing the amount of ductile component. By comparing the molecular structures of the individual first network and second network gels, we found that the true key factor that controls the brittleductile transition is the density of elastically effective polymer strands of the two networks, $v_{\mathrm{e}, 1}$ and $v_{\mathrm{e}, 2}$. When $v_{\mathrm{e}, 2} / v_{\mathrm{e}, 1}<1$, the second network fractures right after the fracture of the first network, and the gels are brittle. When $v_{\mathrm{e}, 2} / v_{\mathrm{e}, 1}>1$, only the first network fractures. As a result, the brittle first network serves as sacrificial bonds, imparting toughness of DN gels. This result provides essential information to design tough materials based on the double network concept.
\end{abstract}




\section{Introduction}

Double network hydrogels or, DN gels, are regarded as one of the most robust synthetic hydrogels. ${ }^{1}$ The tough DN gels have a very special interpenetrating network (IPN) structure. They are comprised of a rigid and brittle $1^{\text {st }}$ network of densely-cross-linked short chains in dilute concentration, and a soft, stretchable $2^{\text {nd }}$ network of loosely-cross-linked long chains in high concentration. The soft second network is densely packed and interpenetrated in the cage of the first network's rigid skeleton, as illustrated in Scheme 1. This special contrasting structure makes the DN gels extra-ordinarily tough, significantly different from conventional IPN gels. The typical tough DN gels, referred to as PAMPS/PAAm DN gels, are synthesized using poly(2acrylamido-2-methylpropane-sulfonic acid) (PAMPS) as the first network and polyacrylamide (PAAm) as the second network. ${ }^{1}$ The PAMPS/PAAm DN gels show high extensibility ( 2,000\% of original length), ${ }^{1}$ strength (tensile fracture stress $\left.\sim 5 \mathrm{MPa}\right)^{2}$ and toughness (fracture energy $\left.\sim 1,000 \mathrm{~J} / \mathrm{m}^{2}\right) .{ }^{3,4}$ Later, many studies have shown that the $\mathrm{DN}$ concept is universal and is applicable to various species of polymeric materials, whenever the above
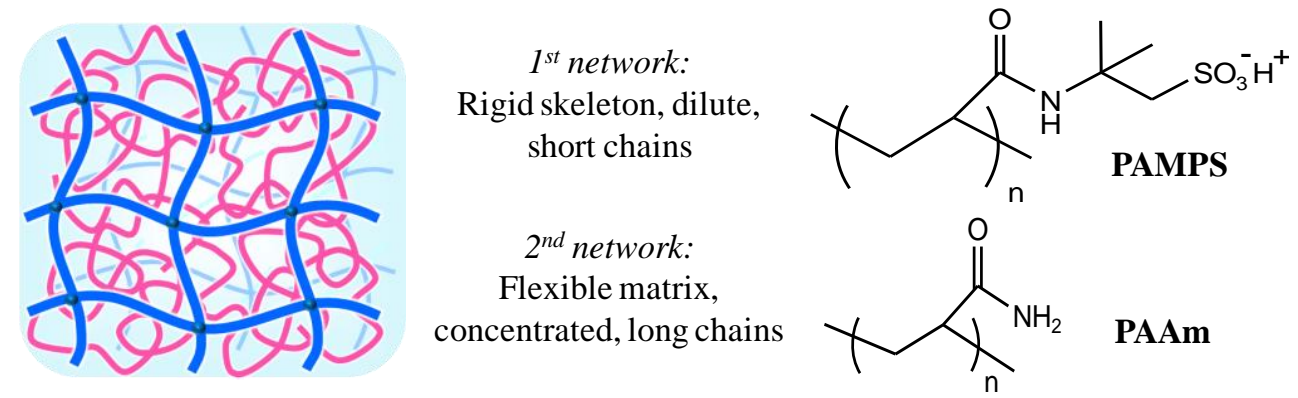

Scheme 1: Illustration of super-tough double network (DN) gels with a contrasting structure. The blue and pink lines represent the $1^{\text {st }}$ and $2^{\text {nd }}$ networks respectively and the filled circles indicate chemical cross-linking points. The two networks do not have internetwork chemical bond, creating a truly interpenetrating network (IPN) structure. The chemical structures of PAMPS and PAAm as first and second networks, respectively, is shown on the right. 
mentioned contrasting DN structure is formed. ${ }^{5-9}$

Many experimental and theoretical studies have been performed to reveal the toughening mechanism of the DN gels. ${ }^{10-13}$ These studies have revealed that the high toughness of DN gels derives from the internal fracture of the brittle network during deformation, which is observed by yielding, necking, and irreversible softening (the Mullins effect) phenomena upon tensile deformation. The internal fracture of the brittle network delocalizes the stress at the crack tip and dissipates a large amount of energy, which substantially retards the crack growth and therefore toughens the material. Previous studies ${ }^{1,14}$ have shown that the structural essence of the tough DN gels can be summarized as follows: 1) Short first network strands and long second network strands, i.e., $N_{2}>>N_{1}$, and 2) Low first network concentration and high second network concentration, i.e., $c_{2}>>c_{1}$. Here $N$ stands for the number of repeated monomeric units of polymer strands between two chemically cross-linked points and $c$ indicates the molar concentration of the monomeric units of polymers.

In this study, we take notice on the latter toughening condition $c_{2}>c_{1}$. The sole first network is very brittle, and the DN gels containing large amount of the second network is ductile and tough. Then, how does the brittle gel change into a ductile and tough one by increasing the amount of the second ductile component $c_{2}$ ? In this paper, we keep the first criteria $N_{2} / N_{1} \gg 1$ satisfied and investigate the effect of the two network concentrations $c_{1}$ and $c_{2}$ on the mechanical behavior of the DN gels. For this purpose, we synthesized 3 sets of DN gels. For each set, we use a constant $1^{\text {st }}$ network cross-linker density but various $2^{\text {nd }}$ network concentrations. First, we prepared PAMPS single network gels with 3 different cross-linker densities. As the swelling degree of a polyelectrolyte hydrogel substantially changes with its cross-linker density, this permits us to modulate $c_{1}$ (which is roughly inverse to $N_{1}$ ) only by changing the cross-linker 
density for preparing the $1^{\text {st }}$ network. In each set of the PAMPS samples, we synthesized the second network from different AAm monomer concentration, $c_{\text {AAm }}$ while keeping the chemical cross-linker density of the second network constant at a very small value. This permits us to keep $N_{2}$ as a large constant value, and modulate the concentration of the second network, $c_{2}$ near the transition regions. We characterized the strength and toughness of these DN gels and determined the brittle-ductile transition points against $c_{2}$. To characterize the internal fracture of these samples, we also carried out the cyclic tensile test. By comparing the tensile behavior of individual single network gels, we found that the brittle-ductile transition is strongly related to the polymer strand density ratio of the two networks, $v_{\mathrm{e}, 2} / v_{\mathrm{e}, 1}$, which indicates that the force balance between the two networks acts as the key structural parameter for this transition.

\section{Experimental Section}

\section{Gel Synthesis}

A. Materials. 2-Acrylamido-2-methylpropanesulfonic acid (AMPS) (Toa Gosei Co., Ltd.) was used as received. Acrylamide (AAm) (Junsei Chemical Co., Ltd.) was purified by recrystallizing from chloroform. Both the cross-linker $N, N^{\prime}$-methylenebis(acrylamide) (MBAA) (Wako Pure Chemical Industries Ltd.) and the initiator 2-oxoglutaric acid (Wako Pure Chemical Industries Ltd.) were used as received.

B. Preparation of $\boldsymbol{t}-\mathbf{D N}$ Gel. The first network PAMPS concentration, $c_{1}$, was varied by changing the first network cross-linker density $c_{1 \_ \text {MBAA }}$ and the second network concentration $c_{2}$, by changing the monomer concentration $c_{\mathrm{AAm}}$. Three sets of samples were synthesized. Each set 
contains the same $c_{1 \_ \text {MBAA }}$ but different $c_{\text {AAm }}$. For simplicity, we coded these DN gels as DN $(x / y)$; where ' $x$ ' and ' $y$ ' are $c_{1 \_\mathrm{MBAA}}\left(\mathrm{mol} \%\right.$, with respect to the monomer concentration) and $c_{\mathrm{AAm}}$ (M), respectively. As has already been clarified in our previous works, conventional DN gels typically contain some internetwork bonds between the first and second networks, which are formed by copolymerization of the second network with the first network through un-reacted residual double bonds of its cross-linker. ${ }^{4,15}$ Since truly independent DN gels (labeled $t$-DN gels) that do not contain any covalent bonds between the two networks have simpler structures than conventional DN gels, in this study we prepared $t$-DN gels for analyzing the properties of the DN gels. This time, we used the simple method to fabricate (pseudo-) $t$-DN gels. The $t$-DN gel samples were prepared using two-step sequential polymerization method with a large amount of initiator in the first network polymerization. ${ }^{4}$ First, a glass mold was prepared by sandwiching a silicone rubber spacer (thickness varying from $1 \sim 3 \mathrm{~mm}$ for different experiments) between two similar glass plates. The $1^{\text {st }}$ network precursor solution was prepared by dissolving $1 \mathrm{M}$ of AMPS, 2, 4 or $6 \mathrm{~mol} \%$ of MBAA as cross-linker and $1 \mathrm{~mol} \%$ of 2-oxoglutaric acid as photo-initiator (the molar percentages are respective to the monomer concentration). The solution was then moved to an argon blanket, made oxygen-free by shaking and then poured into the pre-made glass molds. After irradiation with UV light of $365 \mathrm{~nm}$ wavelength from both sides for 9 hours in an argon atmosphere, the $1^{\text {st }}$ network gel (PAMPS gel) was obtained. Here, the initiator concentration used was much higher than that used in the conventional DN gels which should inactivate the unreacted residual cross-linkers of the first network. Next, these PAMPS gels were immersed and swollen in the $2^{\text {nd }}$ network precursor solution which contained $0.9 \sim 2 \mathrm{M}$ of AAm, $0.02 \mathrm{~mol} \%$ of MBAA, and $0.01 \mathrm{~mol} \%$ of 2-oxoglutaric acid for 3 days. The thickness of the as-prepared PAMPS gels was fixed at $1 \mathrm{~mm}$ and thicknesses of the swollen PAMPS samples in AAm 
solutions are the same with those in pure water and they are tabulated in Table S1 (Supporting information). The swollen PAMPS gels containing AAm monomers were then sandwiched by two glass plates, wrapped, moved to an argon blanket and again irradiated by $365 \mathrm{~nm}$ UV light from one side for 10 hours. In this way, the $2^{\text {nd }}$ network was subsequently polymerized in the presence of the $1^{\text {st }}$ network and the interpenetrating double network (DN) gels were obtained. Previous studies have implied that amount of internetwork covalent bonds in the DN gels synthesized with these compositions is too small to affect properties of them. ${ }^{4}$ The as-prepared DN gels were then swollen in Milli-Q water for 4-5 days to remove any un-reacted monomer and then used for further tests. The sample codes and compositions used in this work are summarized in Table 1. The final thickness of these DN gels after swelling and the subsequent true concentrations of two networks in the DN gels along with the calculation method are shown in the supporting information section (Supporting Figure S1).

For photo-polymerization purpose, we used UV lamps with an intensity of $\sim 4 \mathrm{~mW} / \mathrm{cm}^{2}$ and the distance of the lamps from the sample were maintained as $\sim 10 \mathrm{~cm}$.

C. Preparation of Single Network Gels: Single network PAMPS and PAAm gels were prepared separately from the same precursor solutions of AMPS (1 M AMPS, 2, 4, 6 mol\% of cross-linker and $1 \mathrm{~mol} \%$ of initiator) and AAm (0.8 2 M AAm, 0.02 mol\% cross-linker and 0.01 mol\% of initiator) respectively as that for synthesizing DN gels. 
Table 1. Sample codes and formulations of samples used in this study.

\begin{tabular}{|c|c|c|c|c|c|c|}
\hline \multirow{2}{*}{$\begin{array}{c}\text { Sample } \\
\text { code }\end{array}$} & \multicolumn{3}{|c|}{$1^{\text {st }}$ network } & \multicolumn{3}{|c|}{$2^{\text {nd }}$ network } \\
\hline & $\begin{array}{c}\text { [Monomer], } \\
c_{\mathrm{AMPS}} \\
(\mathrm{M})\end{array}$ & $\begin{array}{c}\text { [Cross-linker] } \\
c_{1 \_\mathrm{MBAA}} \\
(\mathrm{mol} \%)\end{array}$ & $\begin{array}{c}\text { [Initiator] } \\
(\mathrm{mol} \%)\end{array}$ & $\begin{array}{c}\text { [Monomer], } \\
c_{\mathrm{AAm}} \\
(\mathrm{M})\end{array}$ & $\begin{array}{c}\text { [Cross-linker] } \\
c_{2 \_\mathrm{MBAA}} \\
(\mathrm{mol} \%)\end{array}$ & $\begin{array}{c}\text { [Initiator] } \\
(\mathrm{mol} \%)\end{array}$ \\
\hline $\mathrm{DN}(x / y)$ & 1 & $2,4,6(=x)$ & 1 & $0.8 \sim 2(=y)$ & 0.02 & 0.01 \\
\hline PAMPS & 1 & $2,4,6$ & 1 & - & - & - \\
\hline PAAm & - & - & - & $0.8 \sim 2$ & 0.02 & 0.01 \\
\hline
\end{tabular}

*All the concentrations mentioned are the in-feed concentrations used in the precursor solutions.

** The cross-linker and initiator concentrations indicate their in-feed concentrations in mol\%, with respect to the corresponding monomer concentration.

\section{Characterization}

A. Tensile Test. Equilibrium swollen samples (in water) were used for tensile tests of DN gels whereas as-prepared samples were used for sole PAAm gels. For sole PAMPS gels, both swollen and as-prepared ones were used. All the tensile tests were carried out at $25^{\circ} \mathrm{C}$ in air environment and pre-cut dumbbell shaped gel samples (Figure 1a) standardized as the JISK6251-7 size (length of the thinner portion, $L: 12 \mathrm{~mm}$, width of the thinner portion, $b: 2 \mathrm{~mm}$, thickness, $w$ : 1.5 3 mm) were used. A commercial tensile tester Instron 5965 (Instron Co.) equipped with an advanced video extensometer (AVE) was applied for measuring tensile stressstrain (SS) curves of the samples at a fixed tensile velocity of $100 \mathrm{~mm} / \mathrm{min}$. For DN gels and PAMPS gels, the accurate strain was measured by real-time observation of the distance between two white spots marked on the thin portion of the sample with the video extensometer. Only nominal strain was determined in the case of sole PAAm gels, as their high stretchability exceeded the view range of the video extensometer. The fracture stress, $\sigma_{\mathrm{f}}(\mathrm{Pa})$, was obtained 


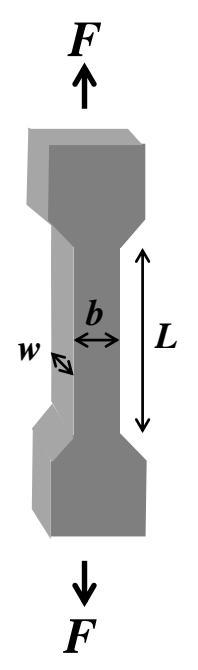

(a)

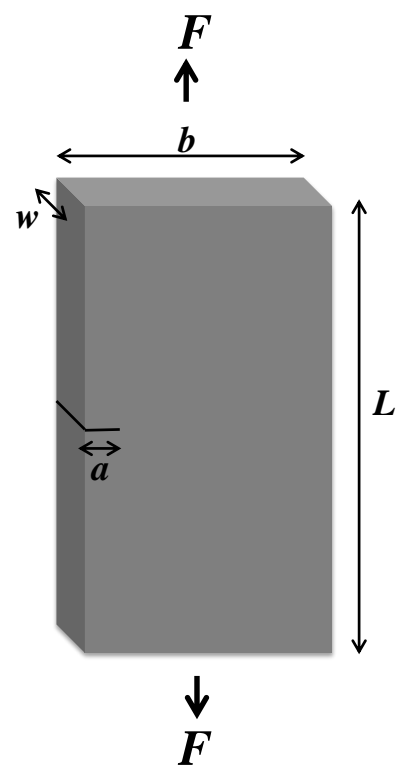

(b)

Figure 1: Illustration of different shapes of gel samples used for the characterization: (a) dumbbell-shaped sample for tensile/cyclic tensile test and (b) bar-shaped sample with a pre-notch for tearing test .

from the nominal stress value at the fracture point. The work of extension to fracture, $W_{\text {ext }}\left(\mathrm{J} / \mathrm{m}^{3}\right)$, which is an indication of toughness of materials, was measured from the area under the SS curve until fracture point. The Young's modulus, E, was determined as the slope of nominal stressstrain curves between the strain range of 0.02 0.2. Each data was the average of 4 measurements, and the error bars were obtained from their standard deviations.

B. Tensile Test with Pre-notched Samples. Tensile test was performed on pre-notched barshaped samples (illustration in Figure 1b) (length, $L=30 \mathrm{~mm}$, width, $b=10 \mathrm{~mm}$, thickness, $w=$ 4 5 mm, initial notch length, $a=1.5 \mathrm{~mm}$ ) with the tensile tester Instron 5965 (Instron Co.) at $25^{\circ} \mathrm{C}$ in the air. One end of the bar-shaped sample was fixed with clamp and the other end was 
pulled at a constant velocity of $100 \mathrm{~mm} / \mathrm{min}$. Crack propagation in mode I (tensile mode of crack opening) was observed as a result of applying force $F$, and the stress intensity factor, $K_{\mathrm{I}}$, ahead of the crack tip was calculated from the following equation, ${ }^{16}$

$$
K_{\mathrm{I}}=\sigma \sqrt{a}\left(1.99-0.41 x+18.7 x^{2}-38.5 x^{3}+53.9 x^{4}\right)
$$

where, $\sigma$ is the stress applied $(\sigma=F / w(b-a)), x$ is the ratio $a / b$. The tearing energy, $T\left(\mathrm{~J} / \mathrm{m}^{2}\right)$, defined as the energy required to fracture a unit surface area in the sample, was calculated as,

$$
T=K_{\mathrm{Ic}}^{2}\left(1-v^{2}\right) / E
$$

where, $K_{\mathrm{Ic}}$ is the critical stress intensity factor for crack propagation, and it was measured from the critical stress at which the pre-notched crack started to propagate, $E$ is the Young's modulus and $v$ is the Poisson's ratio of the soft material (gel). In this paper, we considered the value of $v$ as 0.5 . The fracture energy $T$ thus defined is two times of the energy required to create a unit fractured surface. ${ }^{16}$ Each data was the average of 4 measurements, and the error bars indicate standard deviations.

C. Cyclic Tensile Test. For cyclic tensile test, dumbbell shaped samples standardized as JISK6251-7 size, same as those for tensile test (Figure 1a), were used and Instron 5965 (Instron Co.) tensile tester equipped with AVE was applied to record the successive cyclic tensile stressstrain curves. All the tests were carried out in the air environment at $25^{\circ} \mathrm{C}$. First, the sample was loaded up to a pre-assigned strain, $\varepsilon_{\max }$ and then unloaded until reaching at a strain zero. Then, they were immediately loaded again up to a pre-fixed higher strain and then unloaded. In this way, the same sample was loaded and unloaded up to gradually increasing strain values until 
fracture. As in the case of DN gels, a successive loading curve completely overlaps with the unloading curve of the previous cycle, ${ }^{17,18}$ we consider only the loading curves instead of the unloading curves for simplicity. The dissipated energy, $U_{\text {hys }}\left(\varepsilon_{\max }\right)\left(\mathrm{J} / \mathrm{m}^{3}\right)$, defined as the area enclosed by the first and second loading curves up to the strain $\varepsilon_{\max }$ of the first loading, was calculated as

$$
U_{\text {hys }}\left(\varepsilon_{\max }\right)=\int_{0}^{\varepsilon_{\max }} \sigma_{1} d \varepsilon-\int_{0}^{\varepsilon_{\max }} \sigma_{2} d \varepsilon
$$

where, $\sigma_{1}$ and $\sigma_{2}$ are the nominal stress values for the first and second loading curves, respectively.

\section{Results \& Discussion}

Tensile Behavior of the DN Gels. The tensile stress-strain (SS) curves of DN (2/y), DN (4/y) and DN (6/y) gels are shown in Figure 2(a-c), respectively. When the $2^{\text {nd }}$ network monomer concentration, $y$ is enough high, the DN gels show high fracture stress and fracture strain, and also, the significant 'yielding' phenomenon, which greatly contributes to the excellent toughness of DN gels. ${ }^{10-13}$ These robust DN gels which show this exclusive yielding phenomenon are defined as 'ductile' DN gels. On the other hand, when $y$ is reduced while keeping the same $1^{\text {st }}$ network, the fracture stress and strain of the DN gels suddenly decreases after certain points. For example, in the case of DN (4/y), fracture stress remarkably decreases when $y$ is slightly decreased from 1.1 to 1 . Such DN gels undergo fracture much earlier before yielding appears. Thus, these weak DN gels which do not show yield point are defined as 'brittle' DN gels, and such sudden transformation in fracture parameters has been termed here as 'brittle-ductile transition'. 

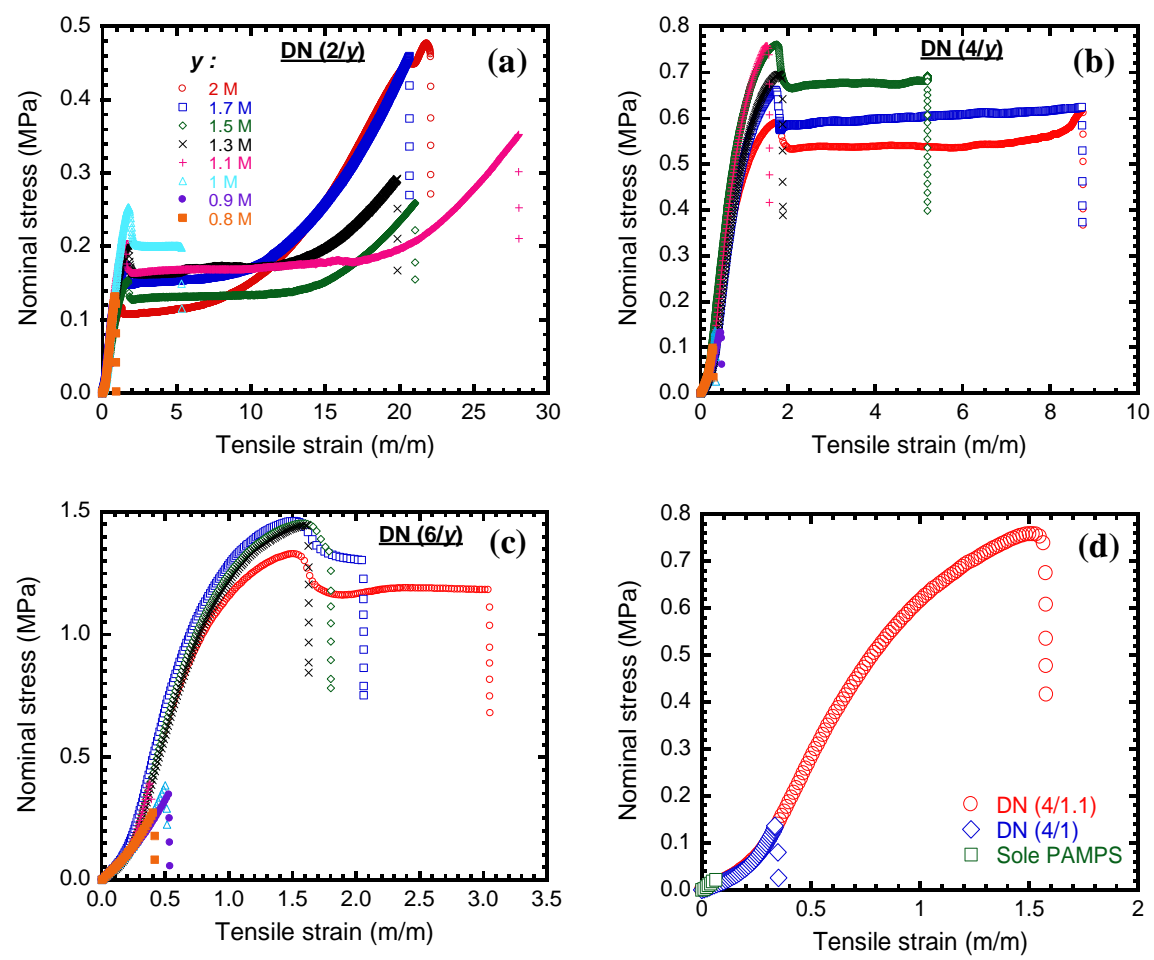

Figure 2: The tensile stress-strain curves of (a) DN (2/y); (b) DN (4/y); (c) DN (6/y) gels and (d) sole PAMPS, DN (4/1) and DN (4/1.1) gels, where ' $y$ ' is the AAm monomer concentration in feed. The symbols and colors for different compositions in (b), (c) are the same as in (a). Tensile stretching rate was $100 \mathrm{~mm} / \mathrm{min}$. Compared to the brittle ones, the other compositions show much higher toughness.

When we compared the tensile SS curves of DN (4/1) (brittle) and DN (4/1.1) (ductile) gels with the sole PAMPS gel (Figure 2d), it is clearly seen from the fracture point that brittle DN gel is only slightly stronger than the sole PAMPS gel which is extremely brittle. The critical $y$ for obtaining ductile DN gels $y_{\mathrm{c}}$ were determined as 1.0, 1.1, and 1.3 M for DN (2/y), (4/y), and (6/y) gels, respectively. We find that as $x\left(1^{\text {st }}\right.$ network cross-linker concentration) increases, which correspond to an increase of PAMPS concentration, $y_{\mathrm{c}}$ also increases. This phenomenon suggests that the brittle-ductile transition is not only governed by one network, rather it is governed by the two networks. 

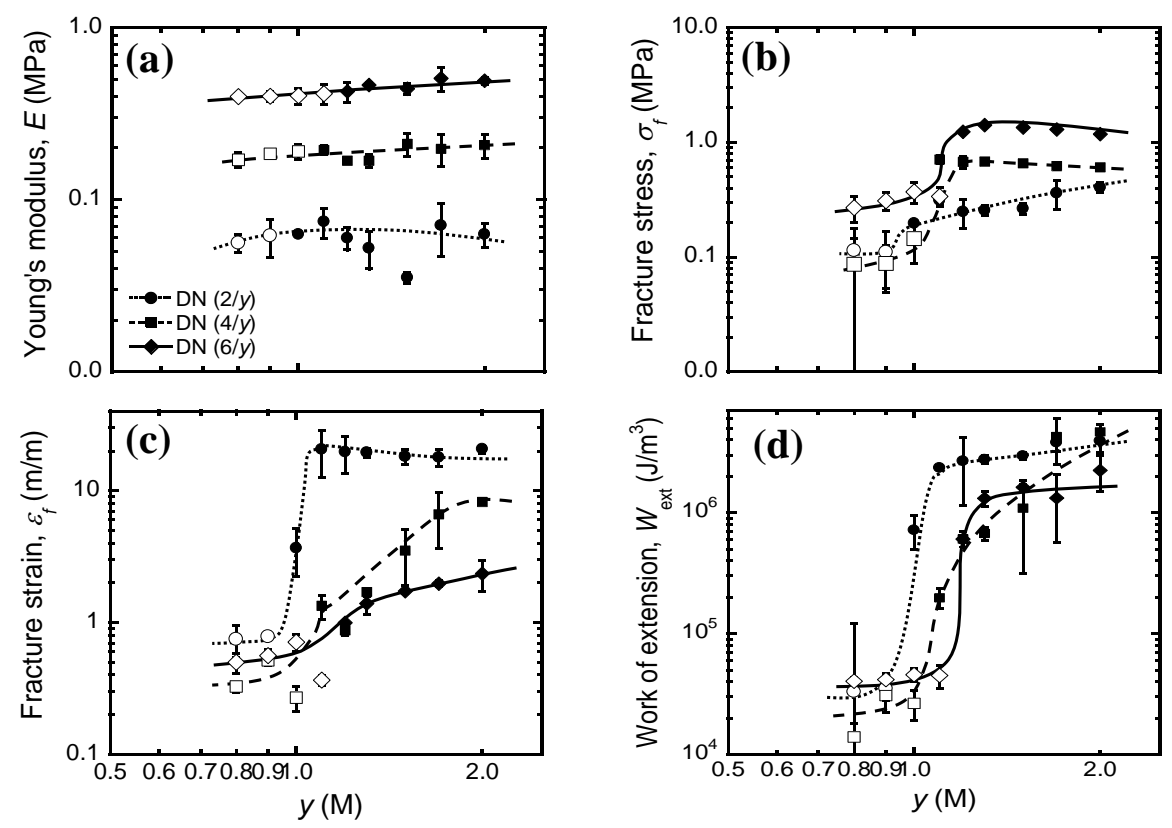

Figure 3: The mechanical toughness characterizing parameters (a) Young's modulus, $E$ (b) fracture stress, $\sigma_{\mathrm{f}}(\mathbf{c})$ fracture strain, $\varepsilon_{\mathrm{f}}$, and (d) work of extension, $W_{\text {ext }}$ against AAm monomer concentration in feed, $y$ in DN (2/y), DN (4/y) and DN (6/y) gels. The closed and open symbols indicate ductile and brittle samples respectively and the symbols in (b)-(d) are the same as in (a). In both cases, brittle samples show much smaller values of the parameters showing very low toughness.

Figure 3 shows the Young's modulus $(E)$, fracture stress $\left(\sigma_{\mathrm{f}}\right)$, fracture strain $\left(\varepsilon_{\mathrm{f}}\right)$, and work of extension ( $\left.W_{\text {ext }}\right)$ against the AAm in feed concentration, $y$ (Figure 3). For a constant set of the PAMPS network, the moduli of the DN gels change very slightly with changing $y$ (Figure 3a). It means that the brittle-ductile transition does not affect the moduli of DN gels. On the other hand, the other parameters were remarkably changed in response to brittle-ductile transition. In terms of the fracture stress, the brittle to ductile transition becomes more abrupt for the sample with high cross-linker density of PAMPS (DN (6/y)) (Figure 3b). In contrast, in terms of fracture strain, we observed an opposite trend (Figure 3c). As a result, the work of extension shows an abrupt transition with $y$ for all the 3 sets of samples (Figure 3d). 
Tensile Behavior of Pre-notched DN Gel Samples. To characterize the toughness, we used uniaxial stretching to pre-notched bar-shaped DN gel samples. ${ }^{19}$ We calculated the tearing energy of both ductile and brittle DN gel samples by estimating the critical stress intensity factor
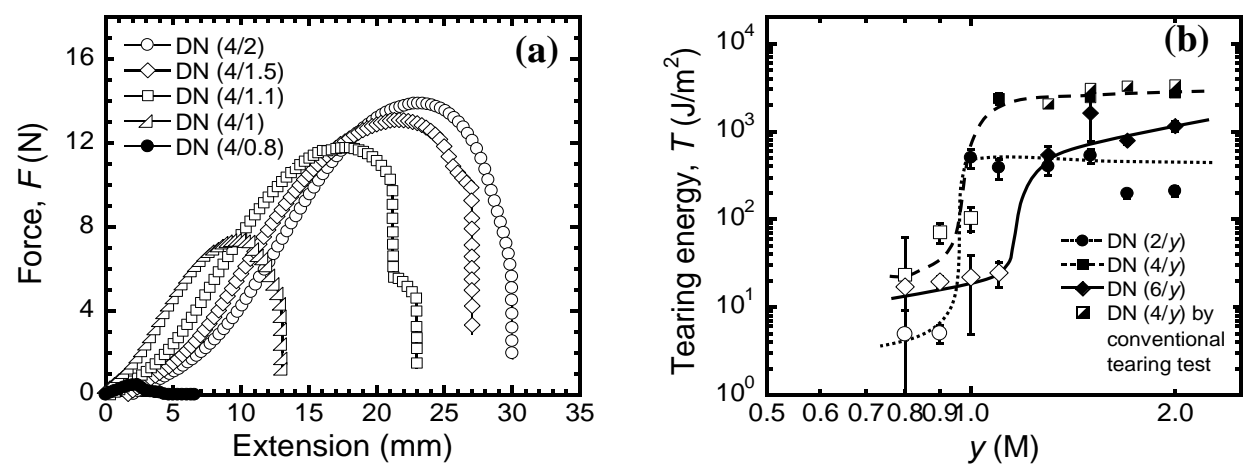

Figure 4: (a) The typical load vs. extension curves for brittle DN (4/0.8), (4/1) and ductile DN (4/1.1), (4/1.5) and (4/2) gels and (b) calculated tearing energy, $T$ values for DN (2/y), (4/y) and (6/y) gels from tearing test. The brittle DN gels (open symbols in (b)) require much reduced force and energy for crack propagation indicating low crack propagation resistance and hence, very small toughness.

for crack propagation $\left(K_{\mathrm{Ic}}\right){ }^{20,21}$ Figure $\mathbf{4 a}$ represents the resulting force vs. extension curves for brittle DN (4/0.8), (4/1) and several ductile DN gel samples. It is obvious from this figure that the applied force at which the pre-notch is converted to a running crack is high in all the ductile DN samples; those are, DN (4/1.1), (4/1.5) and (4/2) compositions; but has a much lower value in the case of the brittle compositions DN (4/0.8) and (4/1). That means, a much smaller force is required to initiate crack propagation in the brittle $\mathrm{DN}$ gel sample, in contrast to the ductile ones.

The tearing energy (T) calculated using Eq. 1 and Eq. 2 for different brittle and ductile compositions is shown in Figure $\mathbf{4 b}$. An abrupt increase in the tearing energy $T$ is also observed 
at critical $y_{\mathrm{c}}$ values, in consistence with the tensile results in Figure 3d. Note that tearing test of a trouser-shaped DN sample with pre-crack ${ }^{22,23}$ has been used to determine gel toughness in our previous studies. ${ }^{3,24}$ This time we could not apply this method for the brittle DN gels because they undergo fracture at the bending point of samples due to their brittle nature. In addition, we have confirmed that the tearing energy values of the ductile DN gels determined by the present method are in agreement with those measured from the tearing test using conventional trousershaped samples ${ }^{19,20}$, as shown in Figure 4b.

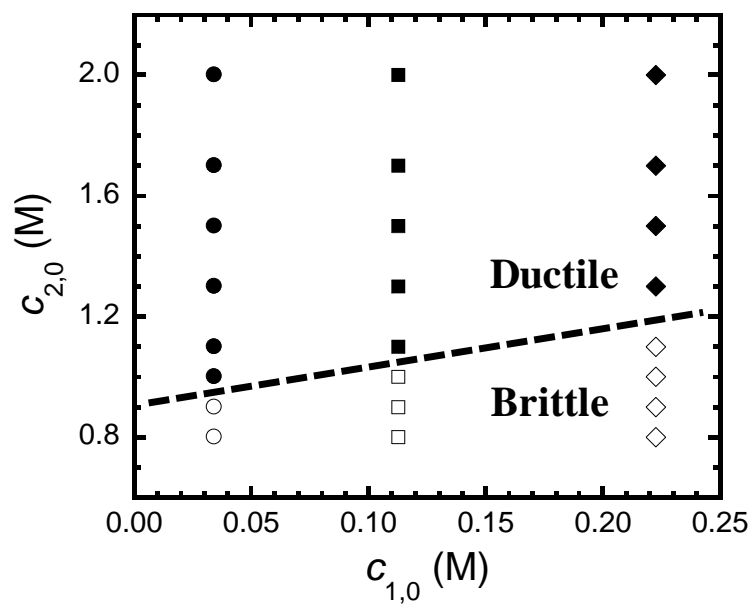

Figure 5: Composition-dependent brittle-ductile phase diagram for different sets of DN gels used in our work. $c_{1,0}$ and $c_{2,0}$ represent the true concentrations of the $1^{\text {st }}$ and $2^{\text {nd }}$ networks in the as-prepared DN gels, respectively. The circles (๑), squares $(\bullet)$ and diamond $(\downarrow)$ shapes indicate the samples DN (2/y), DN (4/y), and DN (6/y), respectively; whereas the filled and open symbols indicate ductile and brittle samples respectively, identified from tensile and tearing test results of their swollen forms.

From the above results, we can construct a mechanical phase diagram in the space of concentrations of the first and second networks (Figure 5). As the entanglement between the two networks depends on the concentrations of the two components at which the IPN structure is 
formed, we adopt the $1^{\text {st }}$ network and $2^{\text {nd }}$ network concentrations, $c_{1,0}$ and $c_{2,0}$, respectively, of the as-prepared IPN samples. Here $c_{1,0}$ was estimated from the thicknesses of the PAMPS gels in their as-prepared and swollen states and $c_{2,0}$ was estimated as $c_{2,0}=c_{\mathrm{AAm}}$, assuming $100 \%$ polymerization efficiency of the precursor monomers ${ }^{25}$ (Supporting Information). Figure 5 shows that the critical $c_{2,0}$ for transition only slightly increases with $c_{1,0}$. The physical meaning of this exclusive phenomenon will be discussed later.

Mechanical Hysteresis Behavior. As has been revealed by the previous studies of our group, the DN gels are toughened by the internal fracture mechanism. This internal fracture of PAMPS remarkably increases the energy for crack growth and delocalizes the stress at the crack tip. As a result, the crack propagation in DN gels is retarded. ${ }^{26,27}$ To relate the brittle-ductile transition to

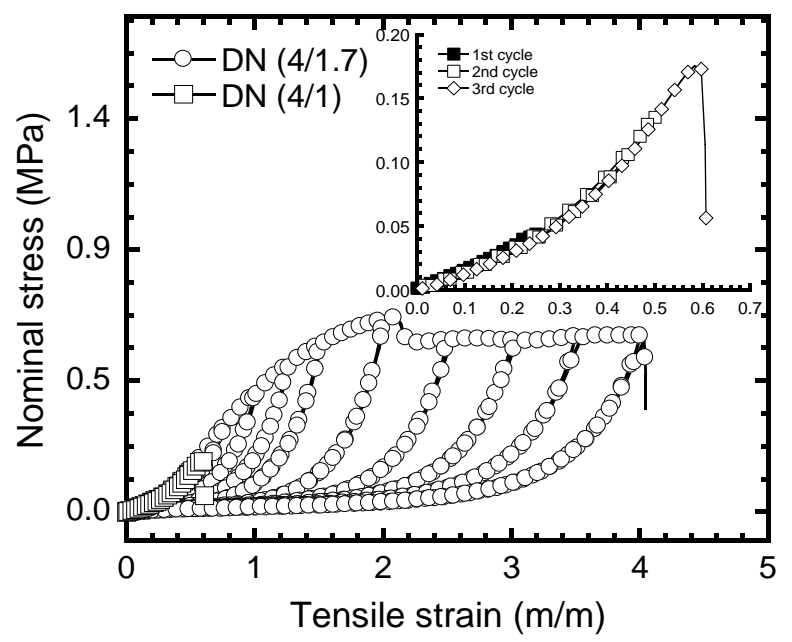

Figure 6: Comparative successive loading curves of brittle DN (4/1) and ductile DN (4/1.7) gels obtained from the cyclic tensile test after applying increasing amount of strain (until fracture). The inset figure provides an enlarged view of the curves of DN (4/1) including different loading cycles which overlap with each other showing almost no hysteresis till sample failure. 
the structure change of the PAMPS network, we further characterized the internal fracture behavior of PAMPS by measuring the hysteresis of the repeated cyclic tensile elongation. The cyclic stress-strain (c-SS) curves of ductile DN (4/1.7) and brittle DN (4/1) gels are shown in Figure 6. The inset figure enlarges the c-SS curves for the brittle sample. For the ductile samples, they show very large amount of hysteresis (i.e. energy dissipation) above a certain strain, and the hysteresis increases with the applied strain until sample breakage. On the contrary, the brittle sample breaks at a very small strain and exhibits almost no hysteresis until breakage. Subsequently, we calculated the amount of dissipated energy from the area of the c-SS curves as a result of applying different amount of strain (Figure 7a) for the brittle (4/1), moderately tough (4/1.5) and the toughest (4/2) DN gels. DN (4/1.5) and DN (4/2) hydrogels initially exhibit very
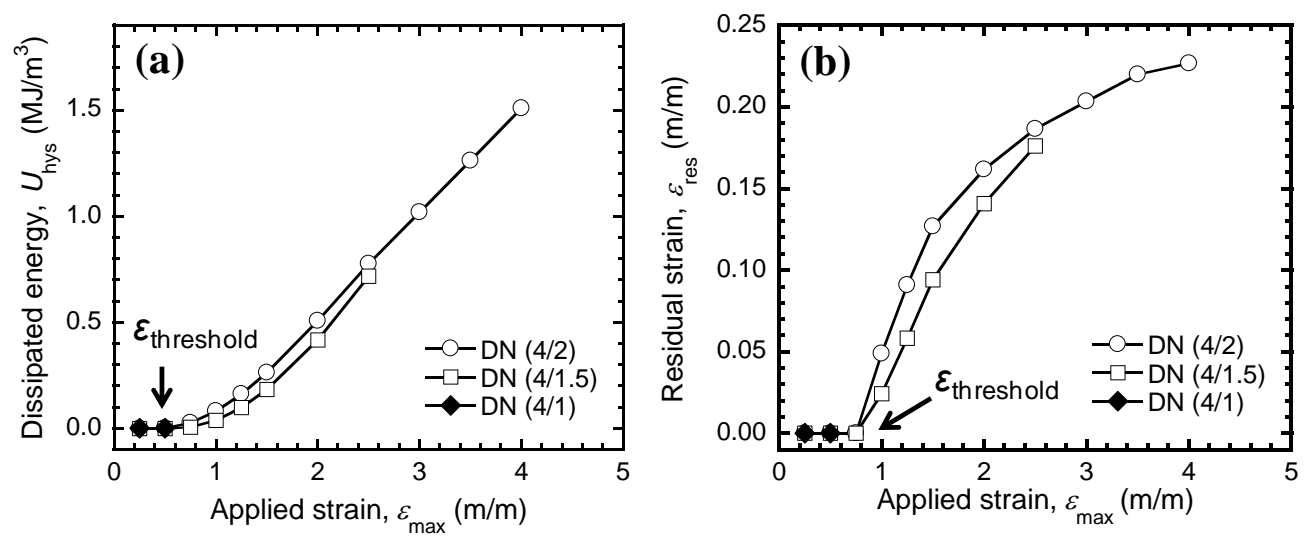

Figure 7: The trend of increasing (a) dissipated energy, $U_{\text {hys }}$ and (b) residual strain, $\varepsilon_{\text {res }}$ as function of the maximum applied strain, $\varepsilon_{\max }$ for brittle DN (4/1), moderately tough (4/1.5) and toughest (4/2) gels. The brittle gel fractures before reaching $\varepsilon_{\text {threshold }}$, without showing $U_{\text {hys }}$ and $\varepsilon_{\text {res }}$. 
little energy dissipation up to a certain strain (we assign it as the 'threshold strain for energy dissipation', $\varepsilon_{\text {threshold }}$ ) and then the energy dissipation increases linearly with increasing strain, in consistence with our recent works. ${ }^{18}$ On the other hand, the brittle DN (4/1) shows almost no energy dissipation and fractures immediately once it reaches the $\varepsilon_{\text {threshold. }}$ Apparently, the $\varepsilon_{\text {threshold }}$ is the point at which the internal fracture of the $1^{\text {st }}$ network starts. For the brittle sample, this starting of the internal fracture leads to propagation of the micro crack and brings sudden global failure of both the PAMPS and PAAm networks. On the other hand, for the ductile sample, the propagation of the $1^{\text {st }}$ network is stopped by the PAAm network. In the latter process, the stress is transferred to a far region behind the crack, which leads to wide-scale internal fracture of the PAMPS network while keeping the structural integrity of the gels.

The internal structural change of materials usually brings about residual strain after deformation. We also investigated the residual strain for different cycles from the c-SS curves for the above-mentioned three compositions (Figure 7b). The brittle DN (4/1) sample that shows elastic behavior exhibits no residual strain until fracture at the $\varepsilon_{\text {threshold. }}$ On the other hand, the ductile samples, DN (4/1.5) and DN (4/2), do exhibit residual strain above the threshold strain $\left(\varepsilon_{\text {threshold }}\right)$, in consistent with the internal fracture of the $1^{\text {st }}$ network. The residual strains, although very small in comparison with the large strains the samples experienced, go through a continuous increase with increasing applied strain. Considering the incompressible nature of gels (Poisson's ratio $v=0.5$ ), these residual strains should bring about a decrease in the cross sectional area of the sample, which can be attributed to the anisotropic fracture of the PAMPS network. ${ }^{18}$

From above results, features of the brittle and the ductile DN gels can be distinguished as follows; the former are brittle, weak, and fracture before catastrophic internal fracture of the $1^{\text {st }}$ 
network whereas the latter are ductile, strong, and fracture after wide-range internal fracture of the $1^{\text {st }}$ network. Therefore, brittle-ductile transition also can be defined by presence or absence of internal fracture.

Mechanical Balance of Two Networks. Then, the question is what determines the occurrence of effective internal fracture? As DN gels consist of two individual networks, it should be governed by the mechanical balance of the two networks. Ideally, this mechanical balance is related to the strength of the two networks, which is determined by their number density of elastically effective polymer strands, $v_{\mathrm{e}, 1}$ and $v_{\mathrm{e}, 2}$. We assume that at the brittle-ductile transition point, $v_{\mathrm{e}, 1}$ and $v_{\mathrm{e}, 2}$ are equal. To confirm this assumption, first, we discuss on $v_{\mathrm{e}, 1}$ and $v_{\mathrm{e}, 2}$ in the as-prepared DN gels.

The $v_{\mathrm{e}, 1}$ of the first network is equal to that of the sole PAMPS gel in swollen state, $v_{\mathrm{e}, 1}^{\mathrm{s}}$. For the $v_{\mathrm{e}, 2}$ of the second soft network in DN gels, we should consider three contributions: chemical cross-linking, trapped entanglement between second network polymer chains, and entanglement of second network polymer chains to the first network. The contribution of internetwork entanglement is also evident in Figure 3c, where we found that $\varepsilon_{\mathrm{f}}$ of DN gels decreases with increasing first network cross-linker density $x$, indicating that the enhanced entanglement of the second network with the first network decreases the extensibility of PAAm chains. The latter two contributions of physical entanglements are predominant in measuring $v_{\mathrm{e}, 2}$ since we used extremely low amount of chemical cross-linker (1 mole of cross-linker for every 5000 moles of monomer) for $2^{\text {nd }}$ network preparation. Assuming that the polymerization of AAm monomers in 
the presence of PAMPS network is the same as that of sole PAAm gel, ${ }^{17}$ the sum of the first and second contributions to $v_{\mathrm{e}, 2}$ can be estimated from the sole PAAm gels $v_{\mathrm{e}, 2}^{\mathrm{s}}$.

Therefore, we investigated the tensile behaviors of individual single network PAMPS gels in swollen state and PAAm gels in as-prepared state, with the same formulation as that of asprepared DN gels. As shown in Figure 8, PAMPS gels are rigid and brittle; thus they fracture at very small strains (nominal fracture strain, $\varepsilon_{\mathrm{f}}<0.35$ ). On the other hand, PAAm gels are soft and

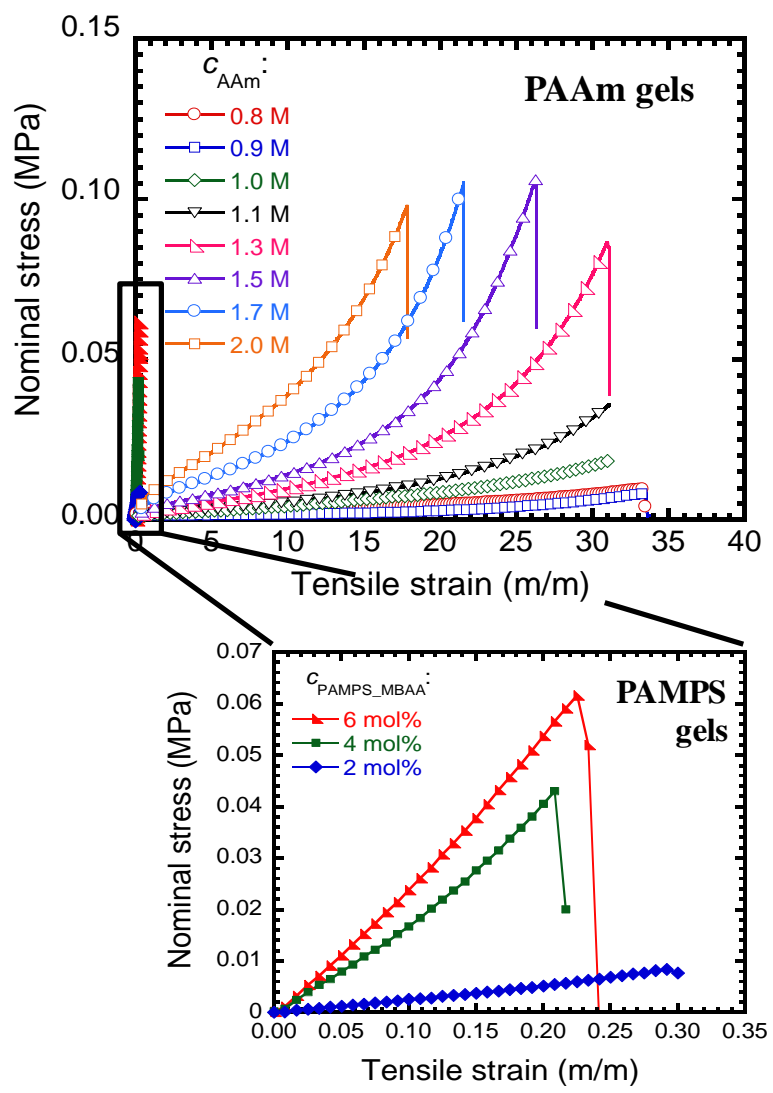

Figure 8: The tensile SS curves of sole PAMPS and PAAm gels having the same concentrations as in their corresponding DN gels. Only a portion of data is shown for a clearer view. Tensile SS curves of sole PAMPS gels are magnified in to a separate figure in the bottom-right for better visibility. 
ductile, showing large fracture strains $\left(\varepsilon_{\mathrm{f}}=15-35\right)$. The $v_{\mathrm{e}, 1}^{\mathrm{s}}$ and $v_{\mathrm{e}, 2}^{\mathrm{s}}$, which are number densities of elastically effective polymer strands of each single network, can be estimated from the Young's moduli of the gels.

Rubber elasticity theories have shown that the number density of elastic strands in Gaussian conformation is related to the Young's moduli of the gels by Eq.4.

$$
E=3 v_{\mathrm{e}} R T
$$

Where $E$ is Young's modulus, $R$ is gas constant, and $T$ is temperature. Since the polymer strands in the neutral gel are in Gaussian conformation, we simply estimated $v_{\mathrm{e}, 2}^{\mathrm{s}}$ from the asprepared PAAm gels by equation 4 (Table S2, supporting information). As the polymer strands of swollen PAMPS gel are not in Gaussian conformation, we could not estimate $v_{\mathrm{e}, 1}^{\mathrm{s}}$ directly from its modulus. Instead, we first estimated the strand density of as-prepared PAMPS gels using 2, 4 and $6 \mathrm{~mol} \% c_{\text {PAMPS_MBAA }}$ and then, we estimated $\nu_{\mathrm{e}, 1}^{\mathrm{s}}$ using the following equation,

$$
v_{\mathrm{e}, 1}^{\mathrm{s}}=\frac{E_{0}}{3 R T}\left(\frac{t_{\mathrm{PAMPS}}^{0}}{t_{\mathrm{PAMPS}}}\right)^{3}
$$

Where, $E_{0}$ is the Young's modulus of the as-prepared PAMPS gels, $t_{\text {PAMPS }}{ }^{0}$ and $t_{\text {PAMPS }}$ are the thicknesses of as-prepared and equilibrium swollen PAMPS gels respectively (Table S1, supporting information).

To elucidate the effect of the network structure on the transition, we first plotted the transition diagram in $v_{\mathrm{e}, 1}^{\mathrm{s}}-v_{\mathrm{e}, 2}^{\mathrm{s}}$ space, without incorporating the internetwork entanglement effect. Except at very small $v_{\mathrm{e}, 1}^{\mathrm{s}}$, the critical $v_{\mathrm{e}, 2}^{\mathrm{s}}$ for transition is not equal to, but lower than $v_{\mathrm{e}, 1}^{\mathrm{s}}$ (Figure 9a). This fact suggests that the third contribution (internetwork entanglement) becomes 

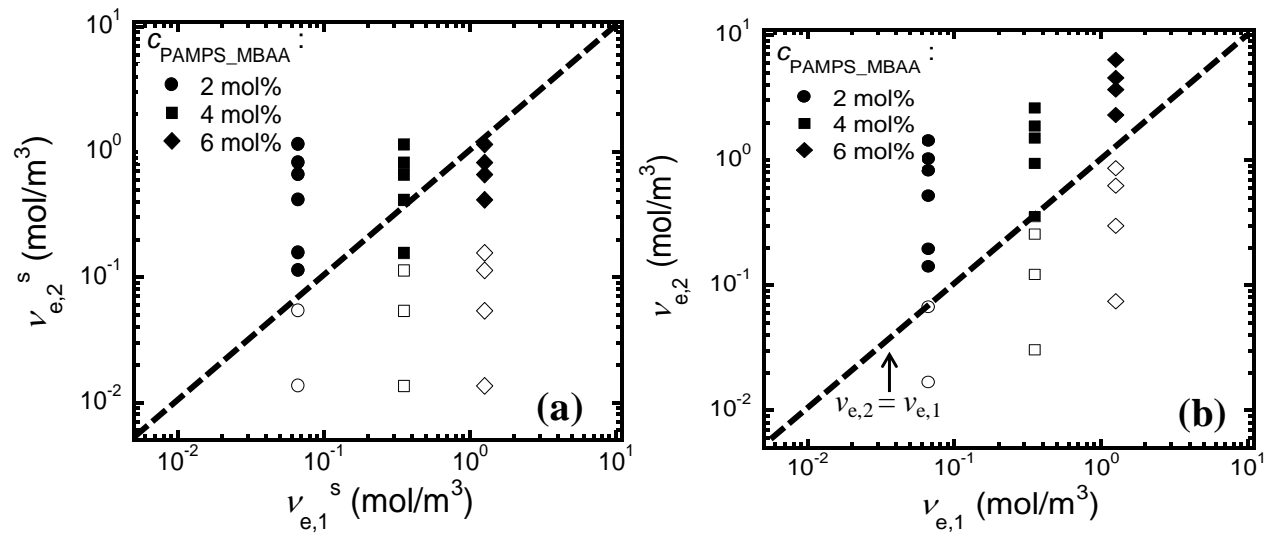

Figure 9: Brittle-ductile phase diagrams in polymer strand density space. (a) $v_{\mathrm{e}, 2}{ }^{\mathrm{s}} \mathrm{vs} . v_{\mathrm{e}, 1}{ }^{\mathrm{s}}$, without considering the internetwork entanglement; (b) $v_{\mathrm{e}, 2}$ vs. $v_{\mathrm{e}, 1}$, after including the correction term for inter-network entanglement for the second network (3.6 $\left.v_{\mathrm{e}, 1}{ }^{\mathrm{s}} v_{\mathrm{e}, 2}{ }^{\mathrm{s}}\right)$. In (b), all the ductile points (filled symbols) are located above the line of $v_{\mathrm{e}, 2}=v_{\mathrm{e}, 1}$, whereas brittle points (open symbols) fall below that line.

important with the increase of $\nu_{\mathrm{e}, 1}^{\mathrm{s}}$ for the transition of DN gels. The $3^{\text {rd }}$ contribution from the entanglement between the two networks should depend on the chain density of both, and the overall $2^{\text {nd }}$ network strand density thus can be expressed as,

$$
v_{\mathrm{e}, 2}=v_{\mathrm{e}, 2}^{\mathrm{s}}+k v_{\mathrm{e}, 1}^{\mathrm{s}} v_{\mathrm{e}, 2}^{\mathrm{s}}
$$

Where $v_{\mathrm{e}, 1}^{\mathrm{s}}, v_{\mathrm{e}, 2}^{\mathrm{s}}$ values are determined from sole networks and $k$ is an unknown constant having a unit of $\mathrm{m}^{3} / \mathrm{mol}$. On the other hand, we can assume $v_{\mathrm{e}, 1}=v_{\mathrm{e}, 1}^{\mathrm{s}}$, since the $1^{\text {st }}$ network is much more rigid than the $2^{\text {nd }}$ network, and hence it's deformation will not be influenced by the entanglement with the $2^{\text {nd }}$ network. Accordingly, we plotted $v_{\mathrm{e}, 2}$ vs. $v_{\mathrm{e}, 1}\left(=v_{\mathrm{e}, 1}^{\mathrm{s}}\right)$ as shown in Figure $9 \mathbf{b}$ and found that by using $k=3.6 \mathrm{~m}^{3} / \mathrm{mol}$, the brittle and ductile data points fall in two different regions separated by a border line $v_{\mathrm{e}, 1}=v_{\mathrm{e}, 2}$. 
This result suggests that the brittle-ductile transition of DN gels is governed by the ratio of the polymer strand densities of the two networks, which is also equivalent to their strength ratio, whereupon the internetwork entanglement plays a very important role. When the PAAm network has higher chain density than PAMPS $\left(v_{\mathrm{e}, 2} / v_{\mathrm{e}, 1}>1\right)$, DN gels become ductile and tough. Conversely, when the chain density of PAAm is lower than that of PAMPS $\left(v_{\mathrm{e}, 2} / v_{\mathrm{e}, 1}<1\right)$, the DN gels are weak and brittle. Thus, we conclude that $\nu_{\mathrm{e}, 1}^{\mathrm{s}}=\nu_{\mathrm{e}, 2}^{\mathrm{s}}$ is the critical condition to determine the brittle-ductile transition.

Two-Spring Model for Brittle-Ductile Transition. The previous experiments and analyses clearly show that the essence of the brittle-ductile transition is the mechanical balance of the two networks. Based on this result, we try to illustrate this brittle-ductile transition in double network gels adopting a two-spring model as shown in Figure 10. The blue and red springs signify the state of the $1^{\text {st }}$ and $2^{\text {nd }}$ network strands in any tiny zone of DN gel, respectively when tensile stress, $\sigma$ is applied on it. For both brittle and ductile gels, the stiff and short PAMPS strands that are in highly extended state fracture first around the threshold strain, $\varepsilon_{\text {threshold }}$ Once this happens, the applied force is transferred to the soft and long PAAm strands that are in coiled state, making them locally stretched. Now, if $v_{\mathrm{e}, 2}>v_{\mathrm{e}, 1}$, these PAAm strands can sustain the force transferred. As the two networks are strongly entangled with each other, in the first case, the stretched PAAm strands also transfer force to PAMPS strands and induce their fracture, which leads to widerange internal fracture of PAMPS, i.e. high toughness of DN gels. Oppositely, if $v_{\mathrm{e}, 2}<v_{\mathrm{e}, 1}$, these PAAm strands cannot sustain the stress and fracture right after the fracture of PAMPS strands. As a result, global sample failure occurs without large energy dissipation. 


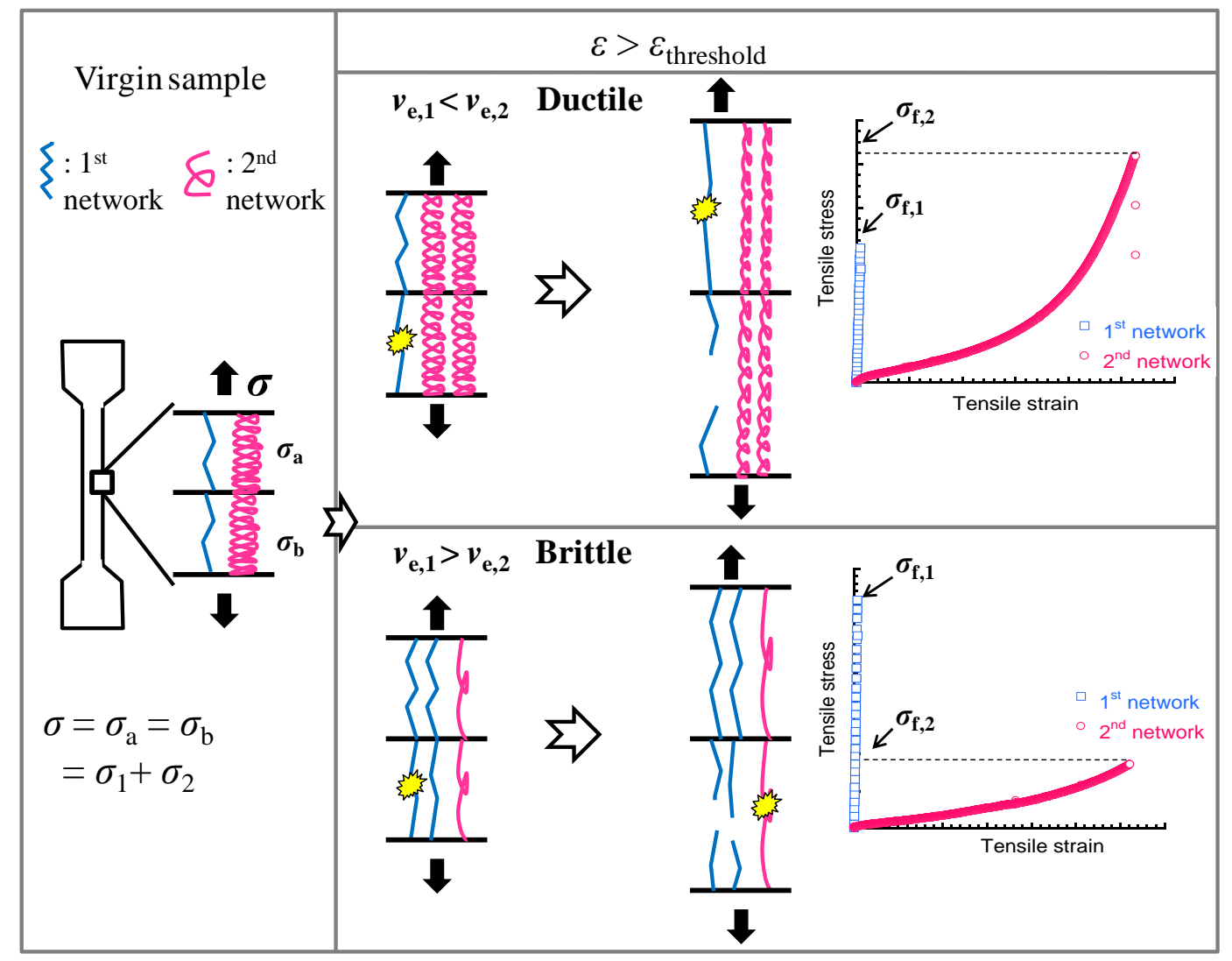

Figure 10: Illustration of the tensile stretching of ductile and brittle DN gels using two springs as model. With stretching, the ductile DN gel (where the effective chain number density of the $2^{\text {nd }}$ network is higher than that of the $1^{\text {st }}$ network) experiences internal damage of the $1^{\text {st }}$ network, while the brittle DN gel (where chain density of the $1^{\text {st }}$ network is higher than that of the $2^{\text {nd }}$ network) undergoes simultaneous fracture of the two networks, which leads to sample failure.

\section{Conclusions}

The composition-dependent brittle-ductile transition of $\mathrm{DN}$ gels is determined by the mechanical balance of the two networks, in which the effective polymer strand density ratio of the two networks, $v_{\mathrm{e}, 2} / v_{\mathrm{e}, 1}$, serves as the key parameter. When the brittle first network has less strand density than the ductile second network $\left(v_{\mathrm{e}, 2} / v_{\mathrm{e}, 1}>1\right)$, the DN gels become tough. Conversely, 
when the brittle network has higher chain density than the ductile network $\left(v_{\mathrm{e}, 2} / v_{\mathrm{e}, 1}<1\right)$, the DN gels become brittle. As the brittle network is comprised of short and extended chains, it always ruptures first, by which the stress is transferred to the ductile network. In the case of $v_{\mathrm{e}, 2} / v_{\mathrm{e}, 1}>1$, the propagation of the micro-crack in the brittle network is stopped by the ductile network, and the latter transfer the stress to a wide region to cause further internal damage of the brittle network. As a result, the brittle network dissipates large amount of energy working as sacrificial bonds and the toughness of the gels is abruptly enhanced. On the other hand, in the case of $v_{\mathrm{e}, 2} / v_{\mathrm{e}, 1}<1$, the ductile network cannot sustain the stress transferred from the brittle network, and it ruptures simultaneously. As a result, the crack propagates catastrophically which leads to an immediate grand failure of the sample to show brittleness. This immediate fracture of PAAm network just after that of PAMPS prevents the internal fracture of the $1^{\text {st }}$ network, thus leads to negligible energy dissipation by brittle samples. Accordingly, a mechanical definition of the DN hydrogels is different from the IPN hydrogels in the sense that DN hydrogels have internal fracture before failure. Except for very low $v_{\mathrm{e}, 1}$ of the first network, $v_{\mathrm{e}, 2}$ of the second network is dominated by internetwork entanglement. The criteria $v_{\mathrm{e}, 2} / v_{\mathrm{e}, 1}>1$ for obtaining tough DN gels is universal and should be used as the key parameter to design tough materials based on the double network concept.

\section{Acknowledgement}

This work was supported by a Grant-in-Aid for Scientific Research (S) (No. 124225006) from Japan Society for the Promotion of Science (JSPS). 


\section{References}

1. Gong, J. P.; Katsuyama, Y.; Kurokawa, T.; Osada, Y. Adv. Mater. 2003, 15, 1155.

2. Kawauchi, Y.; Tanaka, Y.; Furukawa, H.; Kurokawa, T.; Nakajima, T.; Osada Y.; Gong, J. P. J. Phys. Conf. Ser. 2009, 184, 012016.

3. Tanaka, Y.; Kuwabara, R.; Na, Y. -H.; Kurokawa, T.; Gong, J. P.; Osada, Y. J. Phys. Chem. B. 2005, 109, 11559.

4. Nakajima, T.; Furukawa, H.; Tanaka, Y.; Kurokawa, T.; Osada Y.; Gong, J. P. Macromolecules 2009, 42, 2184.

5. Hu, J.; Kurokawa, T.; Hiwatashi, K.; Nakajima, T.; Wu, Z. L.; Liang S.; Gong, J. P. Macromolecules 2012, 45, 5218.

6. Nakajima, T.; Sato, H.; Zhao, Y.; Kawahara, S.; Kurokawa, T.; Sugahara, K. Gong, J. P. Adv. Funct. Mater. 2012, 22, 4426.

7. Suekama, T. C.; Hu, J.; Kurokawa, T.; Gong, J. P., Gehrke, S. H. ACS Macro Lett. 2013, 2, 137.

8. Nakajima, T.; Fukuda, Y.; Kurokawa, T.; Sakai, T.; Chung, U.; Gong, J. P. ACS Macro Lett. 2013, 2, 518.

9. Haiyan, Y.; Akasaki, T.; Sun, T. L.; Nakajima, T.; Kurokawa, T.; Nonoyama, T.; Taira, T.; Saruwatari, Y.; Gong, J. P. J. Mater. Chem. B 2013, 1, 3685.

10. Na, Y. -H.; Tanaka, Y.; Kawauchi, Y.; Furukawa, H.; Sumiyoshi, T.; Gong, J. P.; Osada, Y. Macromolecules 2006, 39, 4641.

11. Webber, R.; Creton, C.; Brown, H. R.; Gong, J. P. Macromolecules 2007, 40, 2917.

12. Brown, H. R. Macromolecules 2007, 40, 3815.

13. Tanaka, Y. Europhys. Lett. 2007, 78, 56005. 
14. Na, Y. -H.; Kurokawa, T.; Katsuyama, Y.; Tsukeshiba, H.; Gong, J. P.; Osada, Y.; Okabe, S.; Karino, T.; Shibayama, M. Macromolecules 2004, 37, 5370.

15. Tominaga, T.; Tirumala, V. R.; Lin, E. K.; Gong, J. P.; Wu, W. -L. J. Phys. Chem. B 2007, 112, 3903.

16. Hertzberg, R. In Deformation and Fracture Mechanics of Engineering Materials, John Wiley and Sons: New York, 1996; p 757.

17. Tsukeshiba, H.; Huang, M.; Na, Y. -H.; Kurokawa, T.; Gong, J. P.; Osada, Y. J. Phys. Chem. B. 2005, 109, 11559.

18. Nakajima, T.; Kurokawa, T.; Ahmed, S.; Wu, W. -L.; Gong, J. P. Soft Matter 2013, 9, 1955.

19. Lin, W. -C.; Fan, W.; Marcellan, A.; Hourdet, D.; Costantino, C. Macromolecules 2010, 43, 2554.

20. Evans, A. G. In Fracture mechanics of ceramics, Plenum, New York, 1974; Vol. 1, p 17.

21. Zarzycki, J. J. Non-Cryst. Solids 1988, 100, 359.

22. Rivlin, R. S.;Thomas, A. G. J. Polym. Sci.1953,10, 291.

23. Greensmith, H. W.;Thomas, A. G. J. Polym. Sci.1955,18, 189.

24. Furukawa, H.; Kuwabara, R.; Tanaka, Y.; Kurokawa, T.; Na, Y. -H.; Osada, Y.; Gong, J. P. Macromolecules 2008, 41, 7173.

25. Kii, A.; Xu, J.; Gong, J. P.; Osada, Y.; Zhang, X. J. Phys. Chem. B 2001, 105, 4565.

26. Tanaka, Y.; Kawauchi, Y.; Kurokawa, T.; Furukawa, H.; Okajima, T.; Gong, J. P. Macromol. Rapid Commun. 2008, 29, 1514.

27. Yu, Q. M.; Tanaka, Y.; Furukawa, H.; Kurokawa, T.; Gong, J. P. Macromolecules 2009, 42, 3852. 
For Table of Contents (TOC) use only

Brittle-Ductile Transition of Double Network Hydrogels: Mechanical Balance of Two Networks as the Key Factor

Saika Ahmed, Tasuku Nakajima, Takayuki Kurokawa, Md. Anamul Haque, and Jian Ping Gong

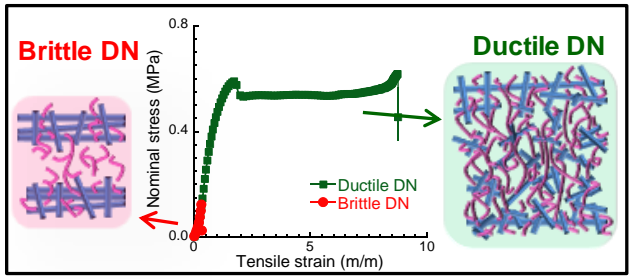

